\title{
Hydroxyapatite-Coated Compaction Short Stem Represents a Characteristic Pattern of Peri- Prosthetic Bone Remodelling After Total Hip Arthroplasty
}

Shinya Hayashi ( $\nabla$ shayashi@med.kobe-u.ac.jp )

Kobe University Graduate School of Medicine

Shingo Hashimoto

Kobe University Graduate School of Medicine

Yuichi Kuroda

Kobe University Graduate School of Medicine

Naoki Nakano

Kobe University Graduate School of Medicine

Tomoyuki Matsumoto

Kobe University Graduate School of Medicine

Tomoyuki Kamenaga

Kobe University Graduate School of Medicine

Takahiro Niikura

Kobe University Graduate School of Medicine

Ryosuke Kuroda

Kobe University Graduate School of Medicine

\section{Research Article}

Keywords: total hip arthroplasty, short tapered-wedge stem, full hydroxyapatite-coated compaction short stem, bone mineral density

Posted Date: January 4th, 2021

DOl: https://doi.org/10.21203/rs.3.rs-137510/v1

License: (9) (1) This work is licensed under a Creative Commons Attribution 4.0 International License. Read Full License 


\section{Abstract}

Purpose: We aimed to investigate the differences in peri-prosthetic bone remodelling between the full hydroxyapatite (HA)-collared compaction short stem and the short tapered-wedge stem.

Methods: This retrospective cohort study enrolled 159 consecutive patients (159 joints) undergoing total hip arthroplasty (THA) using the full HA compaction short $(n=64)$ and short tapered-wedge $(n=95)$ stems. Body mass index (BMI), peri-prosthetic bone mineral density (BMD), and clinical factors, including the Japanese Orthopaedic Association score and the University of California Los Angeles (UCLA) activity score, were assessed and compared.

Results: Both groups showed similar peri-prosthetic BMD changes. Peri-prosthetic BMD was almost maintained in the distal femur and Gruen zone 6 with both type of stems, but significant BMD loss was found in zones 1 and 7 in both groups and in zone 2 of the full HA compaction stem group. Significant positive correlations were found between the proximal femoral BMD changes and the UCLA score in the tapered-wedge stem group but not between BMD changes and clinical factors in the full HA compaction stem group. Femoral bone shape affected the peri-prosthetic BMD changes in the tapered-wedge stem but not in the full HA compaction group. The stem collar of the full HA compaction stem did not affect peri-prosthetic $B M D$, but unique bone remodelling in the calcar region was observed in $27.6 \%$ cases.

Conclusion: Peri-prosthetic bone remodelling remained unaffected by clinical and radiographic factors after THA with the new short full HA compaction stem. Therefore, the new stem may be useful in a variety of cases.

\section{Introduction}

Load bearing of the peri-prosthetic bone after total hip arthroplasty (THA) is critical to maintain bone strength (1). Once a femoral prosthesis has been implanted, daily activities result in mechanical loading of the femoral bone around and below the stem by the implanted stem. However, there are many other factors involved in stress sharing and, consequently, in stress shielding, and these factors influence bone mineral density (BMD) (2). Stress shielding in the femur often occurs in the calcar region because it is highly unlikely that the loading of and stress distribution in the proximal femur will ever be replicated in the presence of an intramedullary prosthesis $(3,4)$, and the decreased BMD may lead to aseptic loosening, stem subsidence, and peri-prosthetic fractures $(3,5)$. Therefore, implants should aim to replicate the physiological stress distribution pattern (6).

Hydroxyapatite $(\mathrm{HA})$ is widely used in THA to improve implant survivorship through accelerated early bone remodelling $(7,8)$. This bioactive coating is believed to enhance the initial fixation by osseointegration $(7,8)$. The ACTIS stem (DePuy Synthes, Warsaw, IN, USA) is a medial-collared, tripletapered short stem. A medial collar provides improved stability, avoiding subsidence with a porous sintered titanium bead coating present proximally and a grit-blasted surface present distally and is entirely HA-coated. It is designed to enhance immediate stability, increase early fixation, and allow easy 
insertion. Further, the implant is inserted by using hybrid of compaction and cutting broach, which enables compaction of the cancellous bone toward the cortical rim until axial and rotational stability is achieved. The cancellous bone is maintained without the implant contacting to the femoral cortex, and the HA-coated femoral stem is inserted into a compacted sleeve of cancellous bone.

Bone-preservation strategies involve the development of neck-preserving short stems designed to avoid stress shielding and increase peri-prosthetic bone formation (9). The Tri-Lock bone preservation (BPS) stem (DePuy Synthes) is a short tapered-wedge stem with a small shoulder shape, short anterior-posterior width, and short length. Short stems potentially preserve more bone stock, which improves proximal load transfer, and show lower stress shielding than long stems $(10,11)$. Maintenance of peri-prosthetic BMD after THA with the Tri-Lock BPS stem has been previously reported (12).

(1) Is there any difference in the peri-prosthetic BMD change after THA between the full HA-collared compaction short stem and the short tapered-wedge stem?

(2) Is there any relation between the pre-operative clinical factors and post-THA BMD change?

(3) Does the stem collar of the HA compaction stem affect peri-prosthetic bone remodelling? Briefly, this study aimed to investigate the differences in peri-prosthetic bone remodelling between the full HAcollared compaction short stem and the short tapered-wedge stem.

\section{Materials And Methods}

\section{Patients' characteristics}

This retrospective cohort study enrolled 159 consecutive patients (159 joints) who underwent THA using the full HA compaction short stem (ACTIS; DePuy Synthes, Warsaw, IN; 64 joints) (Figure 1a) or the short tapered-wedge stem (Tri-Lock BPS; DePuy Synthes; 95 joints) (Figure 1b) at our institution between January 2016 and April 2017 (tapered wedge) and April 2017 and November 2018 (full HA compaction). The pre-operative diagnoses were osteoarthritis (grade 4 according to the Tönnis classification) (114 joints), avascular necrosis of the femoral head (30 joints), and rheumatoid arthritis ( 15 joints). Patients with a distorted anatomy of the proximal femur, osteoporosis (lumbar spine BMD $<0.8$ ), metabolic bone disease, and bilateral THA were excluded.

All procedures were performed via the mini-anterolateral supine approach by a single senior surgeon. Full weight-bearing was allowed for all patients a day after the operation. Body mass index (BMI) was assessed at the time of the operation. The post-operative follow-up included dual energy X-ray absorptiometry (DEXA) scanning and evaluation of clinical factors, including hip function, which was evaluated using two grading methods: (a) the Japanese Orthopaedic Association (JOA) score, which allocates 40 points for pain, 20 points for range of motion, 20 points for walking ability, and 20 points for activities of daily living, with a maximum total score of 100 points (13) and (b) the University of California Los Angeles (UCLA) activity score, which describes subjects' level of activity from 1 ("no 
physical activity, dependent on others") to 10 ("regular participation in impact sports"). The JOA and UCLA scores were evaluated 2 years post-operatively.

\section{DEXA measurements}

DEXA scanning was performed using a DPX-L scanner (GE Lunar Corporation, Madison, WI, USA). DPX-L total body scans were performed using the software version 1.35, which set the appropriate transverse speed at $16 \mathrm{~cm} / \mathrm{s}, 8 \mathrm{~cm} / \mathrm{s}$, or $4 \mathrm{~cm} / \mathrm{s}$ depending on the subject's height. Patients were positioned supine with their leg in the neutral position and a knee and foot support to facilitate the scanning of the anteriorposterior projection of the proximal femur, including the area distal to the prosthesis, using an edgedetection technique. BMD was determined pre-operatively and peri-operatively in seven regions of interest based on the Gruen zones (14). The values were expressed as area BMD in grams per square centimetre. The BMD around the stem was assessed within 1 month post-operatively (baseline BMD) and 6, 12, 18, and 24 months post-operatively. BMD ratios were calculated by dividing each BMD value at $6,12,18$, and 24 months post-operatively by the BMD value obtained within 1 month post-operatively.

\section{Statistical analysis}

All data are expressed as mean \pm standard deviation (SD) unless otherwise indicated. The differences in patients' backgrounds between the full HA compaction and tapered-wedge groups were analysed using an unpaired t-test (Table 1). The frequency of the Dorr types between the two groups was analysed using the chi-square test (Table 2). Sequential changes in BMD within each group were analysed via one-way analysis of variance using Tukey's post-hoc test. Additionally, the BMD at each timepoint was compared between the groups using an unpaired t-test (Table 3). Correlations between peri-prosthetic BMD changes and the clinical factors (age, BMI, UCLA score) in the two groups were analysed using Pearson's correlation coefficient (Table 4). The differences in BMD changes among Dorr types were analysed via one-way analysis of variance using Tukey's post-hoc test (Table 5). To analyse the influence of collarcalcar contact on the peri-prosthetic BMD changes, we compared these changes based on whether the full HA compaction stem collar was in contact with the femoral calcar region or not. The differences in the BMD changes between the stem collar-calcar contact and non-contact groups were analysed using an unpaired t-test (Table 6). A p-value $<0.05$ was considered statistically significant. Post-hoc power analysis was performed using $G^{*}$ Power 3 (15). For a sample size of 64 and 95 among three Dorr classifications and a type-l error ( $\alpha$ ) of 0.05 (one-way ANOVA), the study is expected to provide a power (1- $\beta$ ) of 0.80 and 0.93 for detecting large effect sizes (0.4) with respect to the endpoint of periprosthetic BMD changes at 24 months postoperatively, in both full HA compaction and tapered- wedge groups respectively. For the unpaired t-test, we calculated the effect size by means and SDs based on Hedges' $\mathrm{g}$ for each parameter and the $95 \%$ confidence interval (Cl) for effect sizes (16).

\section{Ethics}

The study protocol was approved by the institutional review board on September 8, 2011 (No. 1220), and informed consent for participation in the study was obtained from all participants. 


\section{Results}

\section{Patients' characteristics}

The mean patient age at operation was $66.2 \pm 10.4$ years. At the clinical evaluation performed 24 months post-operatively, the mean BMI was $23.8 \pm 3.7 \mathrm{~kg} / \mathrm{m}^{2}$, JOA score was $95.0 \pm 7.0$, and UCLA score was 6.3 \pm 1.5 . We divided the patients into two groups: the full HA compaction short-stem group and the tapered-wedge stem group. Thigh pain did not occur at 24 months after THA in the full HA compaction group but occurred in $18.9 \%$ (18/95) of patients in the tapered-wedge stem group. The mean values of the parameters in each group are shown in Table 1; they were not significantly different between the groups except the occurrence of thigh pain.

Table 2 demonstrates the characteristics of femoral bone shape according to the Dorr classification. There was no significant difference in the characteristics of femoral bone shape between the groups.

\section{Peri-prosthetic BMD changes were similar between the groups}

Minimal BMD changes were found in the distal femur (Gruen zones 3, 4, and 5) and zone 6 of both type of stems (Table3). However, a significant BMD loss was observed in zones 1 and 7 at each timepoint in both groups compared with BMD value obtained within 1 month post-operatively (full HA compaction: zone 1,6 months, $p<0.001 ; 12$ months, $p<0.001 ; 18$ months, $p<0.001 ; 24$ months, $p<0.001 ;$ zone 7,6 months, $p<0.001 ; 12$ months, $p<0.001 ; 18$ months, $p<0.001 ; 24$ months, $p<0.001$ ) (tapered wedge: zone 1 , 6 months, $p<0.001 ; 12$ months, $p<0.001 ; 18$ months, $p<0.001 ; 24$ months, $p<0.001$; zone 7,6 months, $p<0.001 ; 12$ months, $p<0.001 ; 18$ months, $p<0.001 ; 24$ months, $p<0.001$ ) (Table 3 ). Additionally, BMD loss was observed in zone 2 at 12, 18, and 24 months post-operatively in the full HA compaction group (12 months, $p=0.003 ; 18$ months, $p<0.001 ; 24$ months, $p<0.001$ ) (Table 3 ). On comparing the BMD changes between the groups, we found significant differences in the BMD change pattern only in zone 2 at 18 and 24 months post-operatively (18 months; $p=0.008,24$ months; $p=0.041$ ). The BMD change pattern in other Gruen zones was similar between the two groups all through the 2 years (Table 3 ).

\section{Correlations between peri-prosthetic BMD changes and clinical factors}

Significant positive correlations were noted between proximal femoral BMD changes at 24 months postoperatively and the UCLA score in the tapered-wedge group, and the correlation coefficients were 0.214 for zone $1(p=0.046)$ and 0.227 for zone $7(p=0.034)$. However, no correlations were noted between the BMD changes and age, BMI at surgery, or the UCLA score 24 months post-operatively in the full HA compaction group (Table 4).

\section{Correlation between femoral bone shape and peri-prosthetic BMD changes in the two groups}

We compared the peri-prosthetic BMD changes at 24 months post-operatively between the two groups according to the Dorr classification for femoral bone shape. Table 5 shows that significant peri-prosthetic BMD changes were found in zones $4(p=0.002)$ and $6(p=0.043)$ of the tapered-wedge stem group among 
Dorr types $A, B$, and $C$. A post-hoc analysis demonstrated that significant peri-prosthetic BMD changes were found among Dorr types $B$ and $C$ in zones $4(p=0.001)$ and $6(p=0.038)$. However, no significant periprosthetic BMD changes were found in any Gruen zone in the full HA compaction group among Dorr types A, B, and C (Table 5).

\section{Relationship between the stem collar and periprosthetic BMD changes}

There were no differences in any of the Gruen zones between the stem collar-calcar contact and noncontact groups (Table 6).

\section{Full HA compaction stem represents bone remodelling in the calcar region}

Evidence of bone remodelling in the calcar region was observed in $27.6 \%$ (8/29 cases) of cases in which the stem collar did not contact with the femoral calcar region. Figure 2 demonstrates two representative cases. Bone remodelling in the calcar region was observed 12 months post-operatively in both cases.

\section{Discussion}

In this study, similar peri-prosthetic BMD changes were observed between the full HA compaction short stem and tapered-wedge stem; patients' characteristics, including the clinical factors and femoral bone shape, affected the post-THA peri-prosthetic BMD in the tapered-wedge stem group but not in the full HA compaction group. We further demonstrated that unique bone remodelling in the calcar region was observed in the full HA compaction stem group.

As previously reported, minimal BMD changes after THA were observed in the distal femur with the shorttapered stem, that is, in Gruen zones 3, 4, and 5, However, significant BMD loss was observed in zones 1 and 7 at 24 months post-operatively (17). In this study, the peri-prosthetic BMD loss observed with the full HA compaction short stem was almost maintained in the middle and distal femur, that is, in Gruen zones $3,4,5$, and 6 , but significant BMD loss was found in Gruen zones 1, 2, and 7 at 24 months postoperatively, and the change in BMD was comparable to that seen with the short-tapered stem, except in Gruen zone 2. Recently, Slullitel et al. conducted a randomized controlled trial demonstrating that BMD reduction in the calcar and proximal lateral regions after THA was smaller with the conventional full HA compaction stem than with the short tapered-wedge stem (18). This result is inconsistent with our results, which revealed that regarding BMD preservation in the proximal femoral region, the results with the newly full HA compaction short stem are similar to those seen with short tapered-wedge stem. One of the reasons for BMD loss in the newly full HA compaction short stem may be dependent on broaching during femoral canal preparation. The broach of newly full HA compaction short stem is hybrid broach of compaction with antero-posterior aspect and cutting with medial-lateral $(\mathrm{ML})$ aspect, and the $\mathrm{ML}$ cutting broaching may cause proximal BMD loss in the newly full HA compaction short stem.

Previous studies have demonstrated a significant correlation between BMD changes in the proximal femur and the activity levels in the triple-tapered stem and short-tapered wedge stem $(12,19,20)$. We 
demonstrated a significant correlation between BMD changes in the proximal femur and the UCLA activity scores in the short tapered-wedge stem group, but no such correlation was found between BMD changes and age, BMI, or the UCLA scores in the full HA compaction short-stem group. These results suggest that daily activities may contribute to femoral stem fixation in the short tapered-wedge stem; however, none of the clinical factors affected femoral stem fixation in the full HA compaction short stem.

The occurrence of thigh pain impairs the restoration of hip function, occasionally resulting in revision surgery; however, this is a common complication following $\operatorname{THA}(21,22)$. Thigh pain was previously reported in $16.7 \%$ of 222 patients after THA using the short tapered-wedge stem (23), and thigh pain was reported by $18.9 \%$ of 95 patients with the short tapered-wedge stem in this study. In contrast, thigh pain did not occur in 133 patients with the conventional full HA compaction stem at the 11.5-year follow-up (24), and $83 \%$ of the patients were completely symptom-free at the 20 -year follow-up (25). This study revealed that thigh pain did not occur with the full HA compaction short-stem at the 2-year follow-up, and the result was similar to that seen with the conventional full HA compaction stem.

Several studies have focused on the peri-prosthetic BMD changes and femoral bone shape $(12,26)$. Nakamura et al. investigated the influence of the proximal femoral canal shape on post-operative BMD changes in the femur around a Zweymüller-type stem and showed that the pre-operative morphology of the femoral canal did not affect the change in BMD 2 years post-operatively (26). A previous study also demonstrated that the proximal femoral canal shape did not affect the post-operative BMD change in 65 patients using the short tapered-wedge stem 2 years post-operatively (12). However, our study demonstrated that the femoral canal shape of Dorr type $C$ contributed to peri-prosthetic BMD loss in 95 patients with the short tapered-wedge stem. This result is inconsistent with previous results (12). This discrepancy may be attributed to the statistical power. Previous study may not have been sufficiently powered to analyse BMD differences between Dorr types B and C. In contrast, the proximal femoral canal shape did not affect post-operative BMD changes in the full HA compaction short stem group.

Previous studies have reported unique calcar bone remodelling with the conventional full HA compaction stem $(25,27)$. Bone remodelling in the calcar region was observed in $15 \%$ of cases, and only two cases of grade 3 stress shielding were noted (25); multivariate analysis revealed that femoral remodelling was not influenced to any significant extent by either patient-related or prosthesis-related factors (such as the collar, positioning, and size) (25). Another study reported bone remodelling in the calcar region in $26.9 \%$ cases but calcar lysis in only $7 \%$ cases (27). This study found that calcar bone remodelling was observed in $29 \%$ of all patients using the new full HA compaction short stem. These results indicate that HA may induce osteoinduction in the calcar region of the HA-coated stem.

The limitations of this study were as follows: First, this was not a randomized study. To evaluate the clinical and radiographical outcomes, the analysis of randomized selection for patients is preferable. The background of the patients was also similar in our study. Second, the sample size, with respect to the evaluation of outcomes, was small. 


\section{Conclusion}

This study investigated the differences in peri-prosthetic bone remodelling between the full HA-collared compaction short stem and short tapered-wedge stem. Peri-prosthetic BMD changes after THA were similar between the two groups. The change in BMD was affected by daily activity levels and Dorr type $C$ femoral shape in the short tapered-wedge stem group but was not affected by any factor in the full HAcollared compaction short stem group. Further, the full HA compaction short stem revealed unique bone remodelling in the calcar region. These finding suggest that the new full HA-collared compaction short stem may be useful in a variety of cases such as the patients with Dorr type C.

\section{Declarations}

Competing of interest: The authors have no conflicts of interest to declare that are relevant to the content of this article.

Funding: There is no funding source.

Ethical approval: This research was approved by the institutional review board of the authors' institution, and the study was performed in accordance with the ethical standards as laid down in the 1964 Declaration of Helsinki.

Consent to Participate: linformed consent for participation in the study was obtained from all participants.

Consent to Publish: The authors affirm that human research participants provided informed consent for publication of the images in Figure 2.

Authors Contributions: SHay participated in the study design, drafting of the manuscript, and data collection. SHas carried out data collection and drafting of the manuscript. YK participated in the data collection and drafting of the manuscript. NN participated in the study design and helped to revised the manuscript. TM participated in the data collection and drafting of the manuscript. TK carried out data collection and drafting of the manuscript. TN carried out data collection and drafting of the manuscript. RK participated in the study design and helped to revised the manuscript. All authors read and approved the final manuscript.

Availability of data and materials: The datasets generated during and/or analysed during the current study are available from the corresponding author on reasonable request.

\section{References}

1. Mulholland SJ, Wyss UP. Activities of daily living in non-Western cultures: range of motion requirements for hip and knee joint implants. Int J Rehabil Res. 2001;24(3):191-8.

2. Huiskes R. The various stress patterns of press-fit, ingrown, and cemented femoral stems. Clinical orthopaedics and related research. 1990(261):27-38. 
3. Kroger H, Venesmaa P, Jurvelin J, Miettinen H, Suomalainen O, Alhava E. Bone density at the proximal femur after total hip arthroplasty. Clin Orthop Relat Res. 1998(352):66-74.

4. Engh CA, McGovern TF, Bobyn JD, Harris WH. A quantitative evaluation of periprosthetic boneremodeling after cementless total hip arthroplasty. The Journal of bone and joint surgery American volume. 1992;74(7):1009-20.

5. Furnes O, Lie SA, Espehaug B, Vollset SE, Engesaeter LB, Havelin LI. Hip disease and the prognosis of total hip replacements. A review of 53,698 primary total hip replacements reported to the Norwegian Arthroplasty Register 1987-99. J Bone Joint Surg Br. 2001;83(4):579-86.

6. Pettersen SH, Wik TS, Skallerud B. Subject specific finite element analysis of stress shielding around a cementless femoral stem. Clinical biomechanics. 2009;24(2):196-202.

7. Chen Y-L, Lin T, Liu A, Shi M-M, Hu B, Shi Z-L, et al. Does hydroxyapatite coating have no advantage over porous coating in primary total hip arthroplasty? A meta-analysis. Journal of orthopaedic surgery and research. 2015;10:21.

8. Vidalain JP. Twenty-year results of the cementless Corail stem. International Orthopaedics. 2011;35(2):189-94.

9. Huiskes $\mathrm{R}$, Weinans $\mathrm{H}$, van Rietbergen $\mathrm{B}$. The relationship between stress shielding and bone resorption around total hip stems and the effects of flexible materials. Clinical orthopaedics and related research. 1992(274):124-34.

10. Molli RG, Lombardi AV, Jr., Berend KR, Adams JB, Sneller MA. A short tapered stem reduces intraoperative complications in primary total hip arthroplasty. Clinical orthopaedics and related research. 2012;470(2):450-61.

11. Patel RM, Smith MC, Woodward CC, Stulberg SD. Stable fixation of short-stem femoral implants in patients 70 years and older. Clinical orthopaedics and related research. 2012;470(2):442-9.

12. Hayashi S, Hashimoto S, Kanzaki N, Kuroda R, Kurosaka M. Daily activity and initial bone mineral density are associated with periprosthetic bone mineral density after total hip arthroplasty. Hip Int. 2016;26(2):169-74.

13. Hasegawa Y, Iwata H, Mizuno M, Genda E, Sato S, Miura T. The natural course of osteoarthritis of the hip due to subluxation or acetabular dysplasia. Arch Orthop Trauma Surg. 1992;111(4):187-91.

14. Gruen TA, McNeice GM, Amstutz HC. "Modes of failure" of cemented stem-type femoral components: a radiographic analysis of loosening. Clinical orthopaedics and related research. 1979(141):17-27.

15. Faul F, Erdfelder E, Buchner A, Lang AG. Statistical power analyses using G*Power 3.1: tests for correlation and regression analyses. Behav Res Methods. 2009;41(4):1149-60.

16. Durlak JA. How to select, calculate, and interpret effect sizes. J Pediatr Psychol. 2009;34(9):917-28.

17. Hayashi S, Hashimoto S, Kanzaki N, Kuroda R, Kurosaka M. Stem anteversion affects periprosthetic bone mineral density after total hip arthroplasty. Hip international : the journal of clinical and experimental research on hip pathology and therapy. 2016;26(3):260-4. 
18. Slullitel PA, Mahatma MM, Farzi M, Grammatopoulos G, Wilkinson JM, Beaule PE. Influence of Femoral Component Design on Proximal Femoral Bone Mass After Total Hip Replacement: A Randomized Controlled Trial. The Journal of bone and joint surgery American volume. 2020.

19. Hayashi S, Nishiyama T, Fujishiro T, Kanzaki N, Hashimoto S, Kurosaka M. Periprosthetic bone mineral density with a cementless triple tapered stem is dependent on daily activity. Int Orthop. 2012;36(6):1137-42.

20. Nam D, Salih R, Barrack RL, Nunley RM. An evaluation of proximal femur bone density in young, active patients undergoing total hip arthroplasty at one year postoperatively. Hip international : the journal of clinical and experimental research on hip pathology and therapy. 2019;29(1):51-7.

21. Domb B, Hostin E, Mont MA, Hungerford DS. Cortical strut grafting for enigmatic thigh pain following total hip arthroplasty. Orthopedics. 2000;23(1):21-4.

22. Lavernia C, D'Apuzzo M, Hernandez VH, Lee DJ. Patient-perceived outcomes in thigh pain after primary arthroplasty of the hip. Clin Orthop Relat Res. 2005;441:268-73.

23. Hayashi S, Hashimoto S, Matsumoto T, Takayama K, Niikura T, Kuroda R. Risk factors of thigh pain following total hip arthroplasty with short, tapered-wedge stem. Int Orthop. 2020;44(12):2553-8.

24. Froimson MI, Garino J, Machenaud A, Vidalain JP. Minimum 10-year results of a tapered, titanium, hydroxyapatite-coated hip stem: an independent review. J Arthroplasty. 2007;22(1):1-7.

25. Vidalain JP. Twenty-year results of the cementless Corail stem. Int Orthop. 2011;35(2):189-94.

26. Nakamura S, Minoda Y, Ohta Y, Sugama R, Yamamura K, Ueyama H, et al. Preoperative Morphology of the Proximal Femoral Canal Did Not Affect the Postoperative Bone Mineral Density Change Around the Zweymuller-Type Stem. Orthopedics. 2019;42(5):e449-e53.

27. Jacquot L, Bonnin MP, Machenaud A, Chouteau J, Saffarini M, Vidalain J-P. Clinical and Radiographic Outcomes at 25-30 Years of a Hip Stem Fully Coated With Hydroxylapatite. The Journal of arthroplasty. 2018;33(2):482-90.

\section{Tables}

Table 1 Patient's background

\begin{tabular}{|llll|}
\hline & Full HA compaction & Tapered wedge & p-value \\
\hline Number of patients & 64 (male 15, female 49) & 95 (male 21, female 74) & \\
\hline Age (y.o.) & $65.5 \pm 12.6$ & $66.4 \pm 9.5$ & 0.729 \\
\hline BMI (kg/m²) & $24.1 \pm 4.2$ & $23.8 \pm 3.4$ & 0.726 \\
\hline JOA score (points) & $95.1 \pm 6.8$ & $94.9 \pm 7.2$ & 0.739 \\
\hline UCLA score (points) & $6.3 \pm 1.7$ & $6.3 \pm 1.5$ & 0.852 \\
\hline
\end{tabular}

Table 2 Number of patients with Dorr types 


\begin{tabular}{|llll|}
\hline Dorr type & HA full compaction & Tapered wedge & p-value \\
\hline A & $6(9.4 \%)$ & $3(3.2 \%)$ & 0.087 \\
\hline B & $53(82.8 \%)$ & $84(88.4 \%)$ & 0.277 \\
\hline C & $5(7.8 \%)$ & $8(8.4 \%)$ & 0.937 \\
\hline
\end{tabular}

Table 3 Comparison of BMD changes between Full HA compaction and Tapered wedge stem at 6, 12,18, and 24 months post-operatively in the Gruen zones 


\begin{tabular}{|c|c|c|c|c|c|c|c|}
\hline & & HA full compaction & Tapered wedge & P-value & Hedges'g & $95 \% \mathrm{Cl}$ & \\
\hline \multirow[t]{4}{*}{ Zone1 } & $6 m$ & $0.94 \pm 0.07$ & $0.94 \pm 0.10$ & 0.687 & 0.00 & -0.32 & 0.32 \\
\hline & $12 m$ & $0.92 \pm 0.10$ & $0.93 \pm 0.17$ & 0.692 & -0.07 & -0.39 & 0.25 \\
\hline & $18 m$ & $0.90 \pm 0.09$ & $0.95 \pm 0.14$ & 0.100 & -0.41 & -0.73 & 0.29 \\
\hline & $24 m$ & $0.91 \pm 0.07$ & $0.93 \pm 0.11$ & 0.745 & -0.21 & -0.53 & 0.11 \\
\hline \multirow[t]{4}{*}{ Zone2 } & $6 m$ & $0.95 \pm 0.08$ & $0.99 \pm 0.10$ & 0.156 & -0.43 & -0.75 & -0.11 \\
\hline & $12 m$ & $0.94 \pm 0.10$ & $0.99 \pm 0.11$ & 0.076 & -0.47 & -0.79 & -0.15 \\
\hline & $18 m$ & $0.94 \pm 0.08$ & $1.01 \pm 0.13$ & $0.019 *$ & -0.62 & -0.95 & -0.30 \\
\hline & $24 m$ & $0.93 \pm 0.08$ & $1.01 \pm 0.13$ & $0.008^{*}$ & -0.71 & -1.04 & -0.38 \\
\hline \multirow[t]{4}{*}{ Zone3 } & $6 m$ & $1.02 \pm 0.05$ & $1.00 \pm 0.13$ & 0.294 & 0.19 & -0.13 & 0.51 \\
\hline & $12 m$ & $1.03 \pm 0.05$ & $1.02 \pm 0.14$ & 0.383 & 0.09 & -0.23 & 0.41 \\
\hline & $18 m$ & $1.03 \pm 0.07$ & $1.04 \pm 0.15$ & 0.894 & -0.08 & -0.40 & 0.24 \\
\hline & $24 m$ & $1.03 \pm 0.07$ & $1.04 \pm 0.15$ & 0.896 & -0.08 & -0.40 & 0.24 \\
\hline \multirow[t]{4}{*}{ Zone4 } & $6 m$ & $1.03 \pm 0.04$ & $1.00 \pm 0.09$ & 0.064 & 0.40 & 0.08 & 0.72 \\
\hline & $12 m$ & $1.04 \pm 0.05$ & $1.00 \pm 0.06$ & 0.491 & 0.71 & 0.38 & 1.04 \\
\hline & $18 m$ & $1.04 \pm 0.05$ & $1.02 \pm 0.11$ & 0.410 & 0.22 & -0.10 & 0.54 \\
\hline & $24 m$ & $1.04 \pm 0.07$ & $1.02 \pm 0.06$ & 0.445 & 0.31 & -0.01 & 0.63 \\
\hline \multirow[t]{4}{*}{ Zone5 } & $6 m$ & $1.03 \pm 0.06$ & $1.02 \pm 0.10$ & 0.132 & 0.12 & -0.20 & 0.43 \\
\hline & $12 m$ & $1.05 \pm 0.08$ & $1.02 \pm 0.11$ & 0.905 & 0.30 & -0.02 & 0.62 \\
\hline & $18 \mathrm{~m}$ & $1.06 \pm 0.08$ & $1.04 \pm 0.14$ & 0.137 & 0.17 & -0.15 & 0.48 \\
\hline & $24 m$ & $1.05 \pm 0.09$ & $1.05 \pm 0.12$ & 0.728 & 0.00 & -0.32 & 0.32 \\
\hline \multirow[t]{4}{*}{ Zone6 } & $6 m$ & $0.99 \pm 0.11$ & $0.99 \pm 0.13$ & 0.653 & 0.00 & -0.32 & 0.32 \\
\hline & $12 m$ & $0.98 \pm 0.13$ & $0.97 \pm 0.15$ & 0.213 & -0.07 & -0.25 & 0.39 \\
\hline & $18 m$ & $0.99 \pm 0.11$ & $1.00 \pm 0.14$ & 0.895 & -0.08 & -0.39 & 0.24 \\
\hline & $24 m$ & $0.98 \pm 0.12$ & $0.99 \pm 0.12$ & 0.651 & -0.08 & -0.40 & 0.23 \\
\hline \multirow[t]{4}{*}{ Zone7 } & $6 m$ & $0.92 \pm 0.17$ & $0.90 \pm 0.21$ & 0.355 & 0.10 & -0.21 & 0.42 \\
\hline & $12 m$ & $0.87 \pm 0.18$ & $0.90 \pm 0.19$ & 0.504 & -0.16 & -0.48 & 0.16 \\
\hline & $18 m$ & $0.83 \pm 0.16$ & $0.91 \pm 0.21$ & 0.062 & -0.42 & -0.74 & 0.10 \\
\hline & $24 m$ & $0.85 \pm 0.21$ & $0.84 \pm 0.15$ & 0.448 & 0.06 & -0.26 & 0.37 \\
\hline
\end{tabular}


$*: p<0.05$

Table 4 Comparison of mean age, BMI, and UCLA score between Full HA compaction and Tapered wedge stem

\begin{tabular}{|c|c|c|c|c|c|c|c|}
\hline & & \multicolumn{3}{|c|}{ Full HA compaction } & \multicolumn{3}{|c|}{ Tapered wedge } \\
\hline & & age & BMI & UCLA & age & BMI & UCLA \\
\hline \multirow[t]{2}{*}{ zone 1} & correlation coefficient & -0.048 & -0.02 & 0.153 & -0.092 & -0.155 & $.214^{\star}$ \\
\hline & p-value & 0.724 & 0.885 & 0.255 & 0.398 & 0.157 & 0.046 \\
\hline \multirow[t]{2}{*}{ zone 2} & correlation coefficient & -0.165 & 0.144 & 0.058 & -0.063 & -0.023 & 0.055 \\
\hline & p-value & 0.224 & 0.284 & 0.67 & 0.564 & 0.832 & 0.615 \\
\hline \multirow[t]{2}{*}{ zone 3} & correlation coefficient & -0.061 & 0.098 & 0.056 & -0.016 & -0.128 & 0.209 \\
\hline & p-value & 0.655 & 0.469 & 0.679 & 0.881 & 0.244 & 0.052 \\
\hline \multirow[t]{2}{*}{ zone 4} & correlation coefficient & 0.26 & -0.032 & -0.005 & 0.17 & -0.068 & -0.091 \\
\hline & p-value & 0.053 & 0.812 & 0.969 & 0.115 & 0.537 & 0.399 \\
\hline \multirow[t]{2}{*}{ zone 5} & correlation coefficient & 0.083 & -0.08 & 0.045 & -0.03 & 0.145 & -0.18 \\
\hline & p-value & 0.545 & 0.554 & 0.739 & 0.785 & 0.186 & 0.095 \\
\hline \multirow[t]{2}{*}{ zone 6} & correlation coefficient & -0.157 & 0.235 & 0.061 & -0.079 & -0.03 & 0.061 \\
\hline & p-value & 0.247 & 0.078 & 0.654 & 0.469 & 0.788 & 0.573 \\
\hline \multirow[t]{2}{*}{ zone 7} & correlation coefficient & -0.094 & 0.211 & 0.078 & 0.04 & -0.097 & $.227 *$ \\
\hline & p-value & 0.491 & 0.134 & 0.567 & 0.714 & 0.376 & 0.034 \\
\hline
\end{tabular}

$*: p<0.05$

Table 5 Comparison of BMD changing at 24 months among Dorr A, B, and C 


\begin{tabular}{|llllllllll|}
\hline & \multicolumn{3}{l}{ HA full compaction } & \multicolumn{5}{c|}{ Tapered wedge } \\
\cline { 2 - 9 } & Dorr A & Dorr B & Dorr C & P-value & Dorr A & Dorr B & Dorr C & P-value \\
\hline Zone1 & $0.91 \pm$ & $0.92 \pm$ & $0.87 \pm$ & 0.632 & $0.97 \pm$ & $0.93 \pm$ & $0.92 \pm$ & 0.812 \\
& 0.04 & 0.14 & 0.06 & & 0.04 & 0.11 & 0.11 & \\
Zone2 & $0.93 \pm$ & $0.94 \pm$ & $0.94 \pm$ & 0.985 & $0.99 \pm$ & $1.00 \pm$ & $1.11 \pm$ & 0.099 \\
& 0.11 & 0.08 & 0.15 & & 0.06 & 0.13 & 0.11 & \\
Zone3 & $1.03 \pm$ & $1.02 \pm$ & $1.07 \pm$ & 0.314 & $1.00 \pm$ & $1.03 \pm$ & $1.06 \pm$ & 0.068 \\
& 0.06 & 0.06 & 0.09 & & 0.05 & 0.15 & 0.04 & \\
\hline Zone4 & $1.06 \pm$ & $1.04 \pm$ & $1.06 \pm$ & 0.645 & $1.02 \pm$ & $1.09 \pm$ & $1.01 \pm$ & 0.002 \\
& 0.07 & 0.07 & 0.03 & & 0.07 & $0.27 *$ & $0.06 *$ & \\
Zone5 & $1.08 \pm$ & $1.04 \pm$ & $1.03 \pm$ & 0.621 & $1.03 \pm$ & $1.04 \pm$ & $1.03 \pm$ & 0.266 \\
& 0.09 & 0.08 & 0.10 & & 0.06 & 0.14 & 0.07 & \\
\hline Zone6 & $1.03 \pm$ & $0.99 \pm$ & $1.00 \pm$ & 0.705 & $1.09 \pm$ & $1.10 \pm$ & $0.98 \pm$ & 0.043 \\
& 0.10 & 0.13 & 0.07 & & 0.02 & $0.15^{\star}$ & $0.11 *$ & \\
\hline Zone7 & $0.90 \pm$ & $0.88 \pm$ & $0.86 \pm$ & 0.844 & $0.88 \pm$ & $0.84 \pm$ & $0.86 \pm$ & 0.770 \\
& 0.30 & 0.20 & 0.18 & & 0.01 & 0.15 & 0.19 & \\
\hline
\end{tabular}

*: $p<0.05$

Table 6 Comparison of BMD change whether stem collar contacting to femoral calcar or not 


\begin{tabular}{|c|c|c|c|c|c|c|}
\hline & $\begin{array}{l}\text { Stem collar } \\
\text { contact to } \\
\text { the femoral } \\
\text { calcar }(N=35)\end{array}$ & $\begin{array}{l}\text { Stem collar does not contact to the } \\
\text { femoral calcar }(\mathrm{N}=29)\end{array}$ & $\begin{array}{l}\text { p- } \\
\text { value }\end{array}$ & $\begin{array}{l}\text { Hedge's } \\
\text { g }\end{array}$ & $95 \% \mathrm{Cl}$ & \\
\hline $\begin{array}{l}\text { zone } \\
1\end{array}$ & $0.92 \pm 0.16$ & $0.91 \pm 0.07$ & 0.275 & 0.08 & -0.41 & 0.57 \\
\hline $\begin{array}{l}\text { zone } \\
2\end{array}$ & $0.95 \pm 0.08$ & $0.92 \pm 0.09$ & 0.491 & 0.35 & -0.14 & 0.85 \\
\hline $\begin{array}{l}\text { zone } \\
3\end{array}$ & $1.04 \pm 0.07$ & $1.01 \pm 0.07$ & 0.905 & 0.42 & -0.07 & 0.92 \\
\hline $\begin{array}{l}\text { zone } \\
4\end{array}$ & $1.05 \pm 0.07$ & $1.04 \pm 0.06$ & 0.432 & 0.15 & -0.34 & 0.64 \\
\hline $\begin{array}{l}\text { zone } \\
5\end{array}$ & $1.04 \pm 0.11$ & $1.05 \pm 0.07$ & 0.445 & -0.11 & -0.59 & 0.39 \\
\hline $\begin{array}{l}\text { zone } \\
6\end{array}$ & $0.98 \pm 0.13$ & $1.01 \pm 0.12$ & 0.371 & -0.24 & -0.73 & 0.26 \\
\hline $\begin{array}{l}\text { zone } \\
7\end{array}$ & $0.85 \pm 0.19$ & $0.88 \pm 0.22$ & 0.148 & -0.15 & -0.64 & 0.35 \\
\hline
\end{tabular}

Figures 


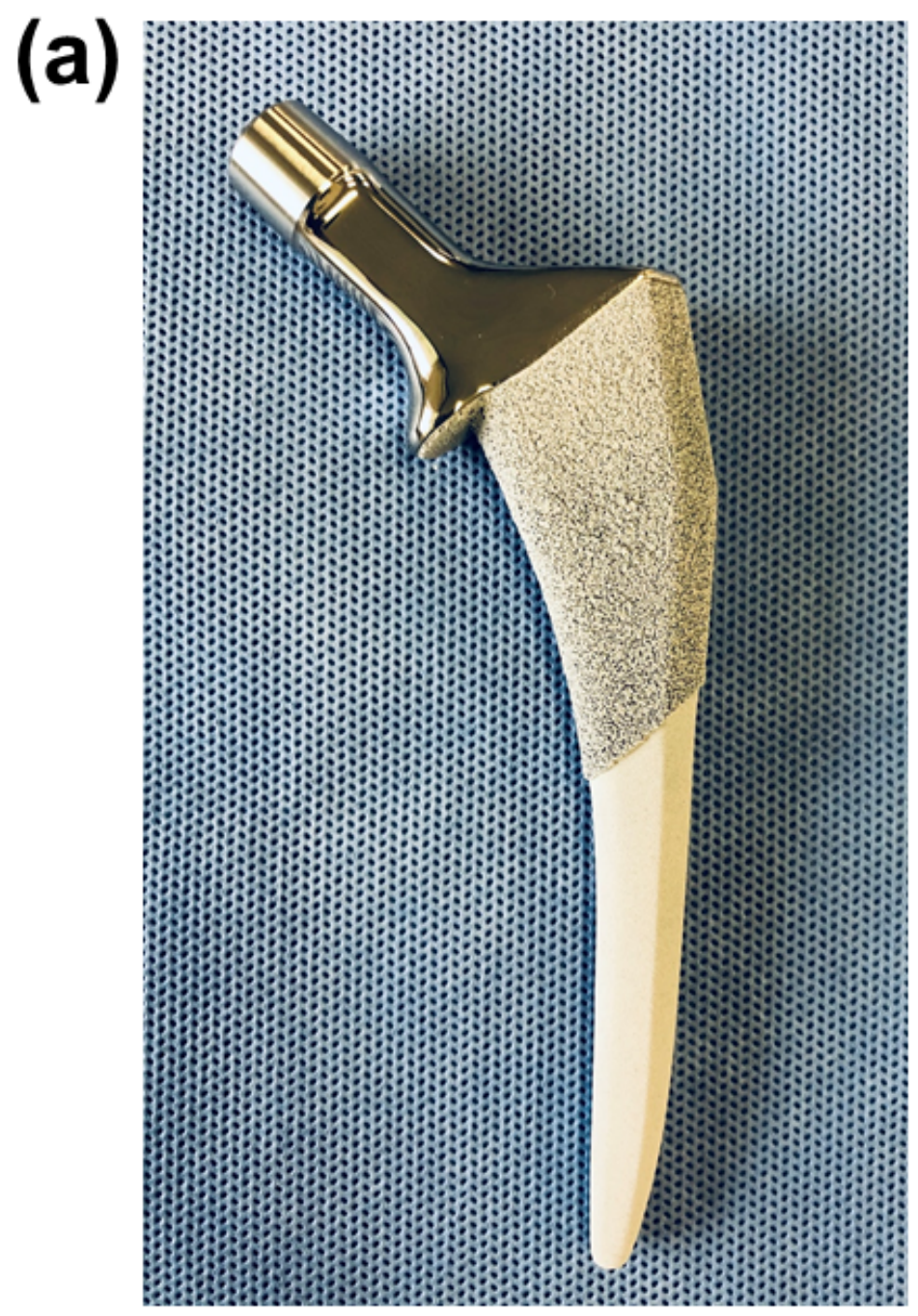

(b)

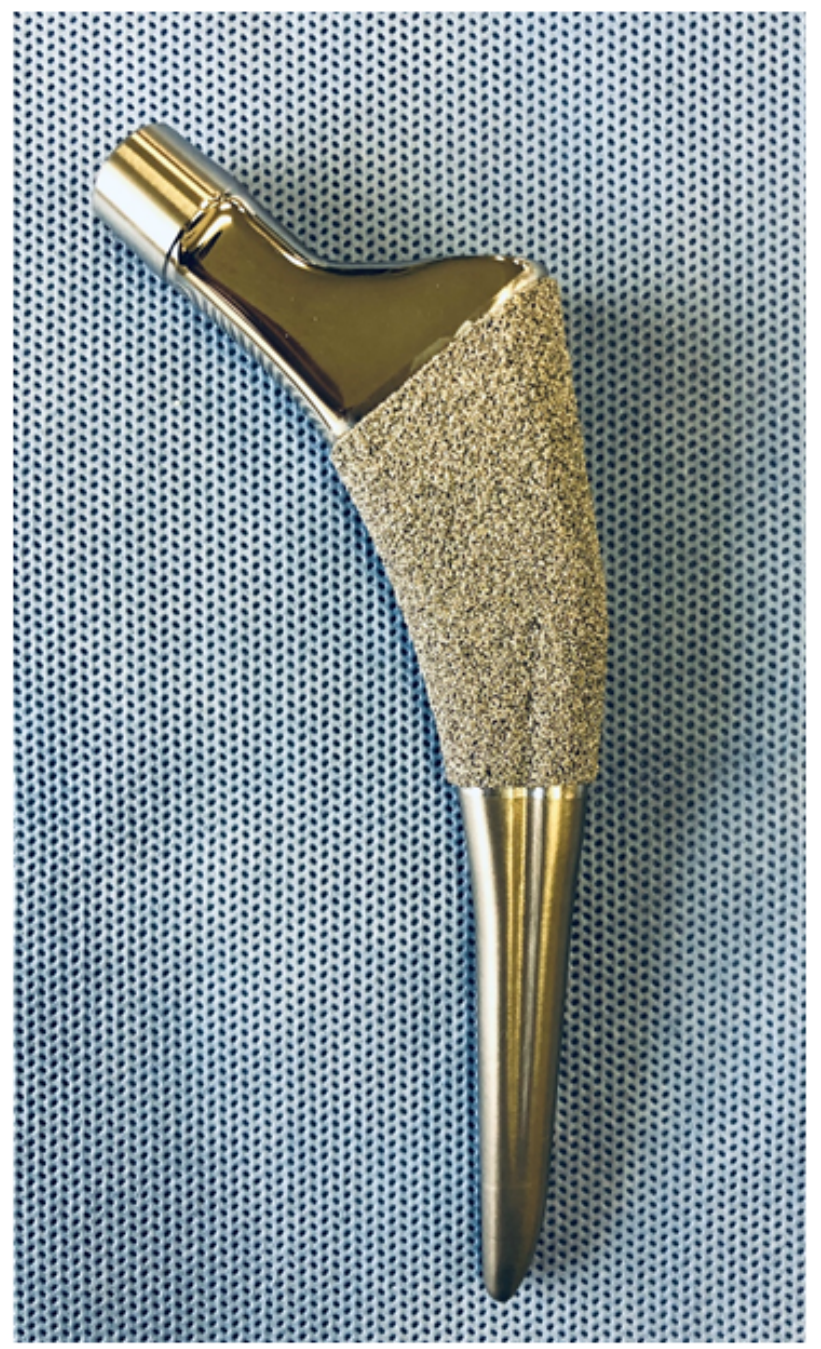

Figure 1

Photographs of (a) the full HA compaction short stem (ACTIS) and (b) the short tapered-wedge stem (TriLock BPS) 


\section{Case 1}

(a)

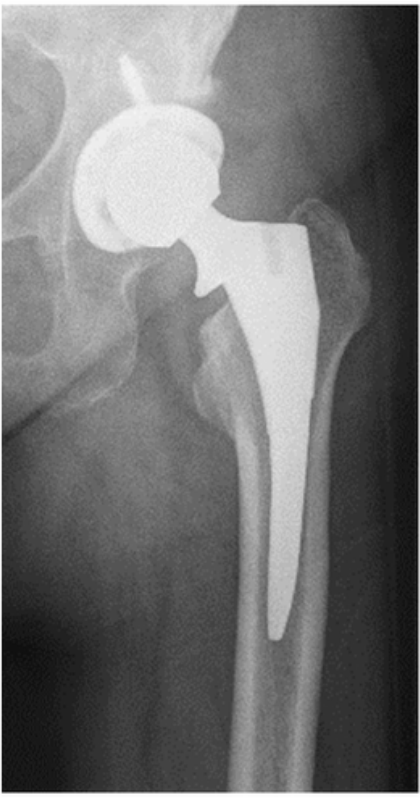

(b)

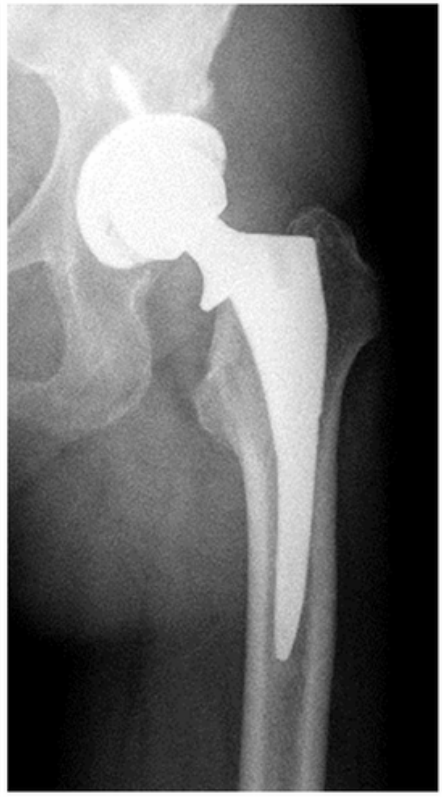

Case 2

(c)

(d)

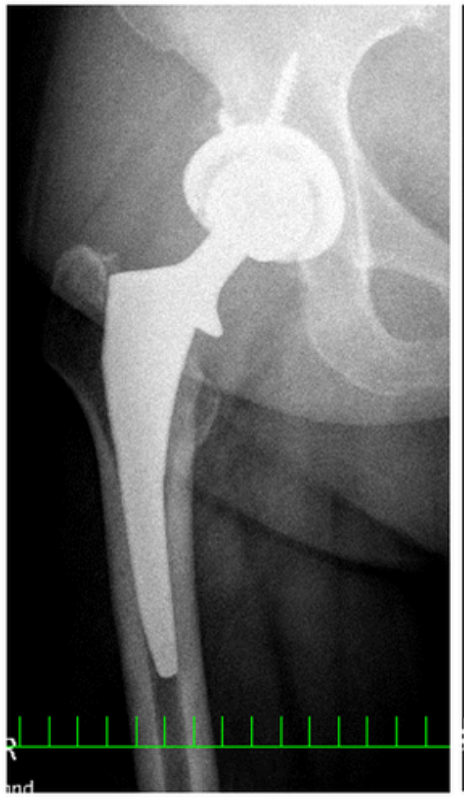

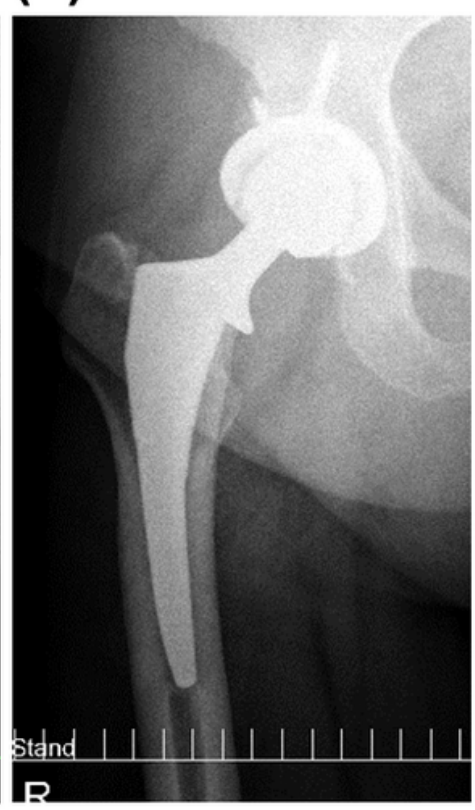

Figure 2

X-ray images (AP view) of two typical cases of bone remodelling in the calcar region with full HA compaction short stem. (a, b) Case 1 and (c, d) Case 2 at (a), (c) initial and (b), (d) 24 months postoperatively 